\title{
La excepción de la excepción. La gobernanza de la migración en tiempos de pandemia
}

\section{The exception of the exception. The migration governance in pandemic times}

Recibido el 9 de diciembre de 2020. Aceptado el 23 de abril de 2021. Publicado el 27 de abril de 2021.

*Autor para correspondencia: Ignacio Irazuzta. Correo electrónico: ignacio. irazuzta@tec.mx

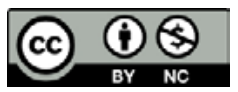

Esta obra está protegida bajo una Licencia Creative Commons Atribución-NoComercial 4.0 Internacional.
Ignacio Irazuzta ${ }^{a^{*}}$ (D) https://orcid.org/0000-0001-8227-9946

Idalia Ibarra ${ }^{a}$ (i) https://orcid.org/0000-0002-2747-1318

\begin{abstract}
a Tecnologico de Monterrey, Escuela de Ciencias Sociales y Gobierno, Monterrey, México, correo electrónico: ignacio.irazuzta@tec.mx, idalia.ib.dlg@gmail.com
\end{abstract}

\section{Resumen}

En este artículo abordamos la gobernanza de la migración en México en el contexto de la pandemia de COVID-19. El objetivo es describir y analizar el funcionamiento de esta forma de gestión, las relaciones entre los actores y las racionalidades que los mueven, así como las transformaciones que la pandemia genera. Observamos específicamente el primer año de pandemia, a través de entrevistas con actores de la gobernanza entre julio y octubre de 2020. Partimos del supuesto de que esta situación excepcional pone en condición de especial visibilidad el complejo entramado de la gobernanza migratoria que opera sobre las fallas en los mecanismos de control de los medios de movimiento de la población migrante. Sobre esta situación, de por sí excepcional de la gobernanza, sostenemos que la pandemia representa un nuevo momento de excepción dadas las restricciones que afectan a aquello que la gobernanza gestiona: la movilidad de poblaciones.

Palabras clave: Gobernanza, migración, pandemia, México.

\section{Abstract}

In this article we address the migration governance in Mexico in the context of the COVID-19 pandemic. The objective is to describe and analyze the functioning of this form of government, the relationship between the actors and the rationalities behind their actions, and the transformations that the pandemic has caused. We specifically analyze the first year of the pandemic through interviews with the actors of the migration governance taking place between July and October 2020. On the basis of the exceptional situation that puts in a special condition of visibility the complex structural framework of the migration governance in that it operates in the failure of the control mechanisms on the transit of the migrant population. We argue that the already exceptional situation of

CÓMO CITAR: Irazuzta, I. \& Ibarra, I. (2021). La excepción de la excepción. La gobernanza de la migración en tiempos de pandemia [The exception of the exception. The migration governance in pandemic times]. Estudios Fronterizos, 22, e068. https://doi. org/10.21670/ref.2105068 
the governance the pandemic represents a new moment of exception given the restrictions that affect the object of this kind of governance: the mobility of populations.

Keywords: Governance, migration, pandemic, Mexico.

\section{Introducción}

La pandemia ha afectado al corazón del fenómeno migratorio en México y más allá de él. Entre las diversas medidas de excepción que han implementado los gobiernos, la de las limitaciones a la movilidad, parcial en México pero más estricta en los países de origen de la migración, es de las principales en cuanto a sus efectos, puesto que ha paralizado o reducido considerablemente el flujo migratorio que se venía dando en el territorio mexicano. Con ello, también las actividades, copiosas y diversas, de gran parte de las organizaciones dedicadas a gestionar el fenómeno como el Alto Comisionado de Naciones Unidas para los Refugiados (ACNUR) que procura la identificación de migrantes huidos de sus países de origen; las instituciones gubernamentales que procesan solicitudes de asilo y retienen a los migrantes en las estaciones migratorias; la amplia red de asistencia de las casas de migrantes que ofrecen ese tipo de refugio temporal que acompasa la travesía por el territorio del país.

Como es sabido, México ha mantenido un ritmo sostenido de migración internacional en tránsito hacia los Estados Unidos que, sorteando incluso la autorización para el ingreso en el país, circula de hecho a lo largo de todo su territorio. En 2019, al menos 788671 migrantes fueron aprehendidos después de cruzar la frontera hacia Estados Unidos (Organización Internacional para las Migraciones [огм], 2020). Esta realidad de hecho ha propiciado el desrrollo de un complejo sistema de gestión de esta migración en tránsito. La llamada "gobernanza de la migración" congrega a multiplicidad de agentes gubernamentales, civiles, nacionales e internacionales que gestionan ese tránsito y que, en esa acción, van dando forma al fenómeno de la migración de una manera excepcional, al atender una emergencia que se entiende como "crisis".

Toda esa gestión se ha visto profundamente afectada por la pandemia a partir de otra serie de medidas gubernamentales centradas en procurar el distanciamiento físico entre los individuos en orden a evitar el contagio. La así llamada "sana distancia" dibuja un panorama de ordenamiento social que entra en franca contradicción con algunas de las prácticas migratorias que regula el sistema de la gobernanza migratoria en el país: ha desmantelado las campañas que implementaba el ACNUR para incitar a los migrantes a solicitar el refugio; ha dejado en suspenso los trámites de esas solicitudes en las instituciones de gobierno dispuestas al efecto como son el Instituto Nacional de Migración [INM] y la Comisión Mexicana para la Ayuda a Refugiados [Comar]); ha redirigido la dinámica de actuación de los consulados de los países de origen de la migración; y, por supuesto, ha afectado severamente la actividad de las ya incontables casas de migrantes, dominadas por la realidad del hacinamiento, que se distribuyen a lo ancho y largo del territorio mexicano. La pandemia ha venido a paralizar de manera repentina todo este denso, complejo y disperso accionar de los diversos agentes que intervienen en la gestión del fenómeno migratorio. Pero el frenazo en seco de toda esa energía actante ha producido efectos que redireccionaron la acción de muchos de estos agentes, ya hechos a la crisis, la emergencia y la urgencia, 
y colocar a algunos en lugares en los que no se hallaban anteriormente al centrar su actuación en atención a necesidades de salud o a requerimientos vitales básicos. La pandemia ha puesto en una situación de excepción a la ya de por sí excepcional gestión de la migración en el país.

En este artículo abordamos la gobernanza de la migración en México en situación de pandemia, que es como decir la excepción de la excepción. Partimos del supuesto, evidenciado luego empíricamente, de que esa situación excepcional pone en condición de especial visibilidad el complejo entramado de la gobernanza migratoria. Dicho supuesto es, no obstante, algo más que intuición. Asume el postulado teórico respecto a que la excepción, como situación en la que lo normal se suspende para ser reemplazado por una realidad gobernada por otras reglas, carga con un especial potencial de visibilidad que exhibe a lo que le preexiste con inusitada elocuencia explicativa y que contiene elementos para una heurística descomunal (Schmitt, 2009). La excepción genera entonces especial apetencia de observación, pero, al mismo tiempo y por la misma razón, tiene como efecto una peculiar e intensa capacidad transformadora. La excepción produce cambio repentino, urgente; deshace y hace con inusual celeridad; hace emerger el hecho que, quizá, dé forma luego al derecho.

Cuando se habla de "la excepción de la excepción" no se está haciendo un juego de palabras con el que se quiera significar una restauración de la normalidad a partir del segundo de los términos; como si, a modo de una deducción aritmética, la adición de negativos produzca un positivo. No es eso. Con la excepción de la excepción se quiere expresar la superposición de capas de anomalías sobre el fenómeno que se analiza. La pandemia, que alude al segundo término de la excepción, se sobrepone a un régimen migratorio - el de la gobernanza de la migración- que es de por sí excepcional en tanto produce y funciona sobre el presupuesto de individuos fuera del reconocimiento del derecho, una suerte de "sujetos imposibles", aunque reales, como se expresa más adelante al usar esta expresión de Ngai (2005). Sobre ese punto de partida de la gobernanza migratoria, la pandemia despliega su excepcionalidad en dos sentidos correlacionados: por un lado, en su afectación a la movilidad y en su disposición del distanciamiento físico, que afecta a esos datos primarios de la acción de migrar y de migrar en las circunstancias y condiciones en las que mayoritariamente se migra por el territorio mexicano; por el otro, porque la pandemia genera esas especiales condiciones de visibilidad sobre el fenómeno, tal como ya se mencionó.

Desde esa premisa, en este artículo, inscrito en un proyecto de investigación colectivo mayor ${ }^{1}$ que responde también a una necesidad excepcional, el objetivo es describir y analizar la gobernanza de la migración en México en el contexto de la pandemia de la COVID-19 problematizando el funcionamiento de esta forma de gestión, las relaciones entre los actores y las racionalidades que los mueven, así como los cambios y las transformaciones que la pandemia genera en todo ese entramado.

\footnotetext{
1 “Transformaciones de la globalización y gestión de la migración. Diagnóstico multidimensional de la situación frente a la pandemia del COVID-19", el cual surge de la convocatoria "Apoyo para proyectos de investigación científica, desarrollo tecnológico e innovación en salud ante la contingencia por COVID-19, convocatoria 2020-1" de Conacyt. Este texto recoge en parte el planteamiento teórico-metodológico de la investigación y un primer análisis empírico de los datos recogidos en las entrevistas realizadas por videoconferencia entre julio y octubre de 2020 , con lo cual, cuando en este artículo se hace referencia a la pandemia, se está aludiendo al primer año de la misma. Pueden consultarse otros productos y avances derivados del proyecto, así como el resto de los integrantes del equipo de investigación en el sitio electrónico: www.poliedrica.mx
} 
Para los propósitos de este trabajo de investigación, el análisis de la gobernanza opera metodológicamente a nivel de las grandes superficies. Es una perspectiva de "gran angular"; intenta abrir el objetivo para procurar una visión mayor, pero recurriendo para ello a técnicas de distancia focal acotadas con el propósito de indagar en la racionalidad de los actores que la constituyen, de la experiencia de su trabajo, de las representaciones de los sujetos que gobiernan. El gran angular corresponde al momento de la proyección, que parte de una idea de la composición de lo que en lenguaje sociológico se denomina campo (Bourdieu, 1997) para, así, seleccionar a sus actores clave. La distancia focal acotada, en cambio, es el momento empírico que se concreta en la técnica de la entrevista en profundidad, con la que se intenta recoger la experiencia de los actores sociales particulares que concurren luego como dato a reconstruir y dotar de significado al conjunto de esos actores, a la gobernanza en general. Para construir esta composición metodológica se analizaron un total de 43 entrevistas con actores involucrados en la gestión de la migración: 28 con organizaciones de la sociedad civil, 10 con organismos gubernamentales y cuatro con organizaciones internacionales. ${ }^{2}$

El artículo se divide en dos grandes apartados. Uno en el que se presentan los presupuestos teórico-conceptuales y se profundiza en la hipótesis que ha guiado la investigación. Allí se plantea un uso sociológico de la noción de gobernanza sin desconocer otras perspectivas que la abordan con intención normativa o desde un enfoque crítico. Seguidamente, se pone esa definición conceptual y metodológica en relación con la situación de pandemia. Se aborda la pandemia como la variable independiente del análisis en tanto que ha venido a afectar al objeto de esa gobernanza fundada sobre el principio del derecho a la libre movilidad de los migrantes. Desde este planteamiento de partida, se pasa al segundo gran apartado dedicado al análisis de los datos empíricos. El interés es destacar dos grandes problemáticas que son analizadas desde la experiencia de los actores de la gobernanza. Por un lado, mediante el apartado titulado "Mapear" se intenta la reconstrucción del campo de la gobernanza según las categorizaciones de los actores que plasma sobre el territorio su acción de gobierno para centrarse luego en la afectación de la pandemia sobre esa forma de gobierno. Por otro, en la sección "Identificar", se analiza una de las acciones fundamentales del campo, la dada por las copiosas y heterogéneas operaciones de registro de los actores de la gobernanza hacia unos sujetos que escapan de las instancias de reconocimiento oficial del Estado a partir de su mayoritaria condición de irregularidad migratoria. El trabajo cierra con unas reflexiones finales que, más que cerrar, abren el camino para futuras rutas de indagación sobre el fenómeno.

\section{La excepción. La gobernanza de la migración en tres perspectivas}

Desde la década de 1990, la noción de gobernanza ha venido consolidándose como un concepto y una práctica dirigida a la gestión de grandes problemas globales. Si se

\footnotetext{
${ }^{2}$ La nomenclatura utilizada en este artículo para hacer referencia a los actores entrevistados corresponde al tipo de actor ("G" para actores gubernamentales, "SC" para organizaciones de la sociedad civil, y "Ol" para organismos internacionales), y a un número aleatorio que representa su número de identificación (ej. E-OI4).
} 
considera que surge como una definición operativa desde un organismo internacional —en informes del Banco Mundial, en 1989 (Launay, 2005, p. 94) - para describir una forma de administración de diferentes actores, su tratamiento en los campos de estudio de la ciencia política y de las relaciones internacionales es de especial importancia, sobre todo en temas que abordan problemáticas sociales con actores tan diversos como lo es la migración. A partir del amplio panorama que se observa en la bibliografía, en este trabajo se destacan dos enfoques, uno normativo y otro crítico. Frente a ello, se propone otro que, con la intención de procurar un acercamiento empírico a esa forma de ejercicio de gobierno, se denomina sociológico.

La perspectiva normativa es la que proviene de las grandes agencias y organizaciones internacionales y de las "comunidades epistémicas" (Shapiro, 2001) que asesoran y guían intelectualmente su accionar. Gobernanza de la migración o migration governance es, así, uno de los términos que figura en el Glosario de la Organización Internacional para las Migraciones. Se dice allí que es "la combinación de leyes y reglamentos, políticas y tradiciones, así como de estructuras organizativas (subnacionales, nacionales, regionales e internacionales) y los procesos pertinentes que dan forma y regulan los enfoques de los Estados con respecto a la migración en todas sus formas, abordando los derechos y responsabilidades y la promoción de la cooperación internacional" (oIM, 2019, p. 138). Se trata de una fórmula de gestión basada en los principios del llamado global governance (Betts, 2011) que, a la vez que procura entender y dar sentido de régimen a las relaciones internacionales que se gestan en un mundo globalizado, intenta normar el ejercicio del poder, desconcentrándolo y distribuyéndolo entre un conjunto de actores para generar entre ellos redes flexibles para la toma de decisiones acerca de asuntos públicos (Pierre, 2000; Porras, 2007; Mayntz, 2000; Natera Peral, 2005) sobre los que se entiende que ningún actor tiene un conocimiento, reconocimiento e información exclusivos sobre el que decidir (Kooiman, 1993). Es decir, la gobernanza sería una forma de gobierno sin autoridad soberana para asuntos que trascienden las fronteras de un estado-nación (Finkelstein, 1995).

Este modo de entender la gestión de la migración y lo que le es propio (las fronteras, la identidad) coincide con el posicionamiento de la migración en un lugar destacado de la agenda internacional desde comienzos del siglo xxi (Domenech, 2018). Se entiende, además, dentro del Marco para la gobernanza de la migración, que la oim publica en 2015 (оIM, 2015). Explícita o implícitamente, tres ideas o principios acerca de la migración alientan esta concepción de la gobernanza. En primer lugar, el discurso de los derechos humanos que asiste a los migrantes y la "razón humanitaria" (Fassin, 2012) que ha de guiar las intervenciones frente al fenómeno de la migración. En segundo lugar, la referencia a lo humanitario se relaciona con la forma de concebir al fenómeno migratorio, entendido como "crisis". Es una crisis signada por la migración irregular, el incremento de la xenofobia y la discriminación, el aumento del negocio de la migración, los problemas en las relaciones bilaterales entre países con cruces migratorios fronterizos y la ruptura de la funcionalidad de las políticas migratorias en relación con las necesidades de mano de obra (Domenech, 2018, p. 117). En tercer lugar, el compromiso por una migración ordenada, regular y segura que terminó consolidándose en el pacto que bajo esos mismos términos se firmó en Naciones Unidas en diciembre de 2018, que no hace más que reconocer el desorden, la irregularidad y la inseguridad de la realidad migratoria mundial para revertirlos mediante la construcción de un nuevo régimen migratorio. 
Más allá de los principios y compromisos de los organismos internacionales, esta versión normativa de la gobernanza migratoria construye la realidad de la gestión del fenómeno pautando el accionar de los actores no solo internacionales, sino también nacionales, civiles, religiosos y gubernamentales hasta darle así forma al fenómeno de la migración sobre el presupuesto incuestionable del "derecho a migrar".

Frente a esta visión normativa e institucional existe una perspectiva crítica. Asentada en una tradición marxista y/o foucaultiana, esta visión concentra su atención en ver la funcionalidad de aquella realidad normativa, bien para poner de relieve la proliferación de violencias y el despojo de condiciones mínimas de existencia de poblaciones frente a la descomposición o exacerbación de los aparatos estatales de monopolio de la violencia y el movimiento (Mbembe, 2011; Varela, 2019, 2020; Slack, 2019), bien para resaltar, como lo hacen Mezzadra y Neilson (2013), el manejo de la movilidad y reproducción de la fuerza de trabajo al insistir en que, frente al colosal empeño de gobernar la migración, se despliegan novedosas formas de lucha de los propios migrantes.

Estos autores entienden que esta manera de gestionar los asuntos de la migración traduce normativamente lo que críticamente había formulado Foucault en sus clases de 1978 como gubernamentalidad. Esa forma desconcentrada y desoberanizada de gobierno que tiene por objeto a la población, a la economía política como saber y a los dispositivos de seguridad como recursos técnicos de control de la misma (Foucault, 1980), es en esencia la forma en la que esta perspectiva entiende a la gobernanza contemporánea de la migración y la administración del régimen de fronteras en la globalización neoliberal. Una gestión flexible de la migración que persigue propiciar los flujos de mano de obra requeridos económicamente y que resulten beneficiosos a una forma de reproducción del capital (Mezzadra \& Neilson, 2013, pp. 176-177). Producto del trabajo de las comunidades epistémicas que van delineando técnicas de control, estándares técnicos y programas de desarrollo de capacidades para dar forma a las políticas migratorias, la gobernanza de la migración se presenta como "un proceso simple de persuasión sin coerción, según los patrones neutrales del cálculo y la gestión del riesgo destacando, a menudo, la "libertad de movimiento de los migrantes" (Mezzadra \& Neilson, 2013, p. 211). Para esta visión de la gobernanza, que la emparenta además a los modos corporativos de la gestión empresarial, los Estados, depositarios tradicionales de la soberanía, siguen siendo los actores principales en la conformación de las políticas migratorias, pero su acción se ve crecientemente confrontada por una multiplicidad heterogénea de actores que juegan un rol crucial y, a veces, impredecible. El acento es puesto sobre el efecto de desoberanización, privatización y multiplicación del poder (Chignola, 2010; Negri, 2010) en orden a favorecer modos flexibles, múltiples y heterogéneos de inserción laboral y, en lo que concierne a las fronteras, más allá de la representación de las mismas como muros, formas igualmente flexibles y desconcentradas que sobrepasan la línea geopolítica hasta alcanzar la acción de cada agente de la gobernanza y la vida de los propios migrantes.

Sin excluir estas perspectivas críticas y normativas de tratar el fenómeno de la gobernanza migratoria, nosotros abordamos la cuestión de un modo sociológico. En este sentido, y bajo una perspectiva institucionalista (Powell \& DiMaggio, 1991; Torpey, 1998), la gobernanza es una práctica de gobierno desarrollada por el conjunto de los actores institucionales, público-estatales, privado-sociales (ONG, asociaciones civiles y religiosas), internacionales, gubernamentales y no gubernamentales, que producen el fenómeno migratorio, en términos colectivos y también individuales; 
producen a la migración y producen al migrante a través de su acción. Lo hacen realizando actividades de control (o dejando de realizarlas); asignando categorías administrativas (migrante legal, irregular, refugiado, solicitante de asilo, etcétera); prestando asistencia y cuidado (de refugio, de defensa de sus derechos); produciendo datos (a través de las comunidades epistémicas que participan en la caracterización del fenómeno o contribuyendo en la ideación de políticas públicas); inscribiendo a los migrantes en un orden de reconocimiento anómalo (ACNUR), etcétera. La migración es el resultado de todo ese entramado de acciones de actores que se erige a partir de esa especial capacidad que, en su forma típica ideal, tienen los estados modernos de aprehender poblaciones y, desde ese mismo acto, excluir a otras determinando así quién es ciudadano y quién no (Torpey, 1998), por lo menos por principio, a partir del derecho, aunque luego en el hecho todos esos actores actúen muy frecuente y mayoritariamente sobre las fallas de ese principio, al menos en el caso que se analiza. Es decir, actúan sobre personas migrantes que, por los motivos que fuere, ajenos o propios de su voluntad, pero siempre justificados en la propia racionalización táctica y estratégica del agente migrante (De Certeau, 2000, pp. 40-44), son esquivos respecto a esa capacidad performativa del Estado de aprehender poblaciones. Son, por lo menos en principio y casi siempre, en su ingreso al territorio nacional, "migrantes sin papeles", "irregulares", "ilegales" (De Genova, 2002) a partir de las propias condiciones que genera la política migratoria y la gestión de la gobernanza. Sobre esos sujetos operan estos agentes y su acción consiste en apresurarse para dotar de papeles e identificar a los migrantes, aunque sea de una manera inestable, precaria, heterogénea y multiplicada. En tanto que fenómeno típico de la globalización, la gobernanza se constituye como uno de los fenómenos de desnacionalización de determinadas capacidades estatales (Sassen, 2006).

La gobernanza es entonces todo ese aparato institucional, en la periferia del Estado y en el Estado mismo, que produce esas poblaciones, en términos colectivos $\mathrm{y}$, paralelamente, en términos individuales, que forja frecuentemente la imagen y el actuar del migrante como subjetividad sufriente o víctima (Gatti, 2017), concebido a partir de su condición de vulnerabilidad (Irazuzta \& Martínez, 2014). En ese sentido nos interesa la gobernanza, desde el papel productor de las instituciones, aunque luego utilicemos también los grandes aportes de la perspectiva crítica y hablemos de la gobernanza como gubernamentalidad foucaultiana (Foucault, 1980; Mezzadra \& Neilson, 2013), es decir, un conjunto de dispositivos, de poderes vivos y actantes, que producen regímenes de verdad en torno a aquello que gobiernan (burocracias, discursos, disposiciones morales, edificios, sujetos). La gubernamentalidad, como la gobernanza tal como la entendemos aquí, es poder "positivo", construye aquello que pretende gobernar y le da forma al fenómeno. Todos los agentes de la gobernanza participan de una biopolítica que gestiona poblaciones (Foucault, 1980; Negri, 2010) y en la que está en juego la vida (y la muerte) de los individuos de esas poblaciones (Agamben, 1998; Fassin, 2018).

En tales condiciones, se caracteriza a la gobernanza de la migración como una situación de excepción. Al hacer uso de este concepto de larga tradición (Schmitt, 2009), retomado y resignificado por Agamben, se entiende la excepción como esa "forma legal de lo que no puede tener forma legal" (Agamben, 2004, p. 24), como ese "vacío de derecho" (Agamben, 2004, p. 95) que genera reglas a partir de una suspensión de la ley por obra del soberano que produce un orden propio fuera del orden. La excepción opera en dos niveles de la gobernanza migratoria. En un nivel estructural que erosiona el principio del 
control monopólico de los medios de violencia por el Estado (Torpey, 1998) y da forma a la gobernanza de la migración en el territorio de México al conformar la racionalidad y la práctica de los actores que en ella intervienen y, en general, producir el fenómeno de la migración a partir de ese conjunto de acciones. En otro nivel, en cuanto a la producción del sujeto migrante, opera esa gobernanza en lo que es consustancial a dicho monopolio, es decir, en las fallas sobre los mecanismos y estrategias de identificación de los sujetos que se mueven.

\section{La pandemia: excepción de la excepción}

Para los efectos de este trabajo, la pandemia de la COVID-19 es la variable independiente. La entendemos como una enfermedad de globalización, puesto que afecta a su sistema nervioso central que está constituido por la movilidad (Urry, 2007). Afecta por tanto al fenómeno migratorio y a la gobernanza de la migración, por lo menos en dos aspectos centrales de la misma. Por un lado, en el accionar de los propios actores, en las condiciones en que lo hacen, en la disposición de los espacios donde actúan. Por el otro, la afectación se cierne en el presupuesto mismo sobre el que opera la gobernanza: el derecho a la movilidad.

Como lo hiciera notar Hannah Arendt (1960), la libertad de movimiento es históricamente la más antigua y la más elemental: su restricción ha sido esencial para el mantenimiento de la esclavitud (Bauder, 2016; Mezzadra, 2020) y su liberación para el desarrollo de las modernas sociedades capitalistas al posibilitar los negocios de su clase hegemónica, la burguesía (Pérez-Agote, 1989), así como también para la conformación de la clase obrera desde las grandes migraciones modernas, nacionales e internacionales (Mezzadra, 2005). Bien es cierto también que esa libertad de movimiento es relativa a los confines del estado territorial y que la migración moderna se constituye a partir de esos confines. Desde la conformación del absolutismo en Europa, el estado se arroga el derecho a autorizar y regular de manera monopólica la entrada y la salida de las personas a su territorio con el desarrollo, paralela y progresivamente, de complejos mecanismos de identificación de las personas sobre el principio de diferenciación básico entre nacionales y extranjeros (Torpey, 1998). Por lo tanto, la libertad de circulación al interior del territorio contrasta con el control y la regulación desde y hacia el exterior.

Este rasgo tan central de los modernos estados-nación es un elemento clave en la constitución de los ensamblajes de territorio, autoridad y derecho que Sassen (2006) utiliza para analizar la emergencia de un orden global que erosiona algunos de los principios constitutivos del Estado-nación. La tensión entre libertad y regulación de la movilidad afecta al territorio porque este se ve jalonado por las poblaciones que lo recorren en su tránsito migratorio y porque desestabiliza sus fronteras, fragmentándolas en cada uno de esos pasos y "desgarrándola de la línea magnética que corresponde a la línea de separación geopolítica entre estadosnación" (Mezzadra \& Neilson, 2013, p. 21). Esos fragmentos y desgarramientos se observan en la figura del mapa de la siguiente sección en la que, acompasando el tránsito migrante, se muestra el despliegue ingente y multiplicado de los agentes de la gobernanza. La gobernanza misma, con su intervención sobre esa autoridad monopólica de los medios de movimiento legítimos del Estado, parece estar 
desensamblando las estructuras de autoridad del Estado-nación, que si bien no pierde el control sobre la determinación de quién porta la condición de nacional y quién la de extranjero, sí pierde el monopolio de registro sobre las personas, como se demuestra en el apartado que sigue. El conjunto mismo de ese entramado de gestión de la población migrante está redefiniendo las estructuras de los derechos subjetivos de formas anómalas a través de reconocimientos y registros múltiples y precarios, como también se muestra en el apartado correspondiente.

Sobre ese entramado inestable de ensamblajes que se caracteriza como excepción en la sección anterior, la pandemia ha venido a ejercer un efecto paralizante. Las restricciones a la movilidad, si bien no detuvieron del todo los flujos, provocaron una disminución notable: tan solo en México, el total de entradas mensuales disminuyó en 58\% con respecto a 2019 (oIM, 2020, p. 5). Esto también se ha visto reflejado en las solicitudes de asilo, las cuales han disminuido en $48.5 \%$ con respecto a 2019 (Comar, 2020a). Si bien la migración no se ha detenido, la pandemia sí ha tenido un efecto ralentizante en la movilidad y ha dejado en situación de inmovilidad a las personas que al momento de establecerse esas medidas se encontraban en el territorio mexicano. Otro de los efectos es que ha invertido algunos de los flujos migratorios de sur a norte al mostrar a algunos migrantes que intentan regresar a sus países de origen. Las percepciones de agentes pertenecientes a la sociedad civil reconocen que al inicio de la emergencia sanitaria el flujo de migrantes se dio "a la inversa", incluso mencionan que estos tenían problemas para ingresar a sus países de origen por las restricciones en el tránsito.

Por otro lado, las medidas de distanciamiento social implementadas por el gobierno han afectado el modus operandi de los actores de la gobernanza. Desde los lugares de refugio o detención, como las casas de migrantes y las estaciones migratorias, caracterizados ambos por el hacinamiento, hasta las instancias administrativas de procesamiento de solicitudes de asilo, pasando por la labor de los consulados en la atención a sus nacionales en tránsito o asentados, la gobernanza se ha visto profundamente afectada en su funcionamiento.

Excepción sobre excepción. Las medidas implementadas a raíz de la pandemia intervienen sobre un régimen de excepción que se edifica sobre las fallas del control monopólico de los medios de movimiento legítimos del Estado-nacional. Y sobre ello, una excepción epistémica que muestra al fenómeno de la gobernanza de una manera inusitada, propicia para inducir a sus actores a una reflexión sobre su cotidianeidad interrumpida o para cavilar sobre los fundamentos de su acción.

El análisis de estas reflexiones recogidas en el trabajo empírico de la investigación se organiza en dos secciones que son fundamentales de la gobernanza migratoria en México. En primer lugar, la titulada "Mapear", en la que a la vez que intenta construir sociológicamente el campo de la gobernanza, muestra el territorio en su propio ejercicio de gobierno. En segundo lugar, "Identificar" exhibe a esos actores en la práctica multiplicada del registro de unos sujetos migrantes que, si bien parecen haber perdido aquello que hace a la condición moderna de sujeto — su identidad civil, no dejan por ello de ser identificados una y otra vez a lo largo de su travesía por el territorio. 


\section{Mapear}

Utilizamos este verbo en dos sentidos. Por un lado, mapear refiere a un momento metodológico de la investigación que se asume como la construcción o reconstrucción del campo (Bourdieu, 1997), es decir, útil para trazar las principales líneas del espacio social sobre el que se despliegan los actores de la gobernanza, con sus racionalidades y sus relaciones en un esquema de distribución del poder que permite cartografiar de manera descriptiva la gobernanza de la migración en el país. Por otro, en el sentido de De Certeau (2000), el verbo remite a la noción de prácticas espacializantes y espacializadas. El mapa intenta así captar las acciones sobre el terreno para mostrar los recorridos y los lugares que constituyen el espacio. Los lugares, como puntos estables de posiciones de los actores, son las manifestaciones concretas de los diferentes ejercicios de gobierno encarnados en cada uno de los agentes de la gobernanza; el espacio es el conjunto de lugares que dibujan sobre el territorio la gobernanza de la migración. Ese espacio es, como para De Certeau, "un cruzamiento de movilidades"; un "lugar practicado", "el efecto producido por las operaciones que lo orientan, lo circunstancian y lo llevan a funcionar como una unidad” (De Certeau, 2000, p. 219). Lo que en este sentido se observa en esta práctica de mapear es al territorio como ese lugar practicado, temporalizado y circunstanciado por agentes que son fragmentos de fronteras, desprendimientos como efecto de su multiplicación en el capitalismo global (Mezzadra \& Neilson, 2013) y de las fallas en el monopolio estatal de los medios legítimos de movimiento (Torpey, 1998).

Desde el primer sentido de mapear, el punto de partida de la investigación agrupó a los actores de ese campo en tres categorizaciones básicas. Por un lado, lo que definimos como actores de la sociedad civil, es decir, organizaciones relativamente al margen de las instituciones de gobierno que se desenvuelven por canales de participación alternativos a los institucionalmente previstos y que actúan sobre los principios de una ciudadanía activa en problemas sociales puntuales y diversos (Olvera, 2001). Como componente de la gobernanza de la migración, el de la sociedad civil es un gran espacio de intervención en el que ubicamos a actores diversos en sus funciones y propósitos.

Un lugar destacado en esta categoría de agentes lo ocupan las casas de migrantes. Estas casas o albergues son los lugares de refugio y recuperación para las personas migrantes en su travesía por el territorio. Las constituye el principio asistencial, muy asociado a la iglesia católica y a su larga tradición de santuario (Washington, 2020; Shoemaker, 2013; Lippert \& Rehaag, 2013; Doering-White, 2018), actualizado en la cultura de los derechos humanos y el humanitarismo (Fassin, 2007) que convoca a muchos de los voluntarios que allí prestan sus servicios. Son lugares de transitoriedad, puesto que en su mayoría ofrecen los servicios por estancias cortas, que oscilan entre los tres y cinco días. Se distribuyen a lo largo del territorio del país, acompasando la travesía, como si el paso organizara los lugares, o como si esos lugares que son los albergues imaginaran el paso a través de sus medios de movimiento y terminaran haciendo la migración que acogen. Albergues y ferrocarril se corresponden en esencia, bien porque tienden a ubicarse en las cercanías de las vías; bien porque el tren es su imaginario constitutivo a través de relatos o de su evocación en los murales que ilustran las paredes de casi todos los albergues. Como sea, tren y albergues hacen al dato primario de la travesía migrante y, en última instancia, al objeto de la gobernanza. La Figura 1, en el trazado de la línea ferroviaria del istmo de Tehuantepec, muestra la 
disposición de estos actores junto a las vías del ferrocarril, como si estas construyeran el itinerario y este tipo de dispositivos que gobierna la migración en tránsito.

Figura 1: Albergues y sistema ferroviario en el istmo de Tehuantepec, México

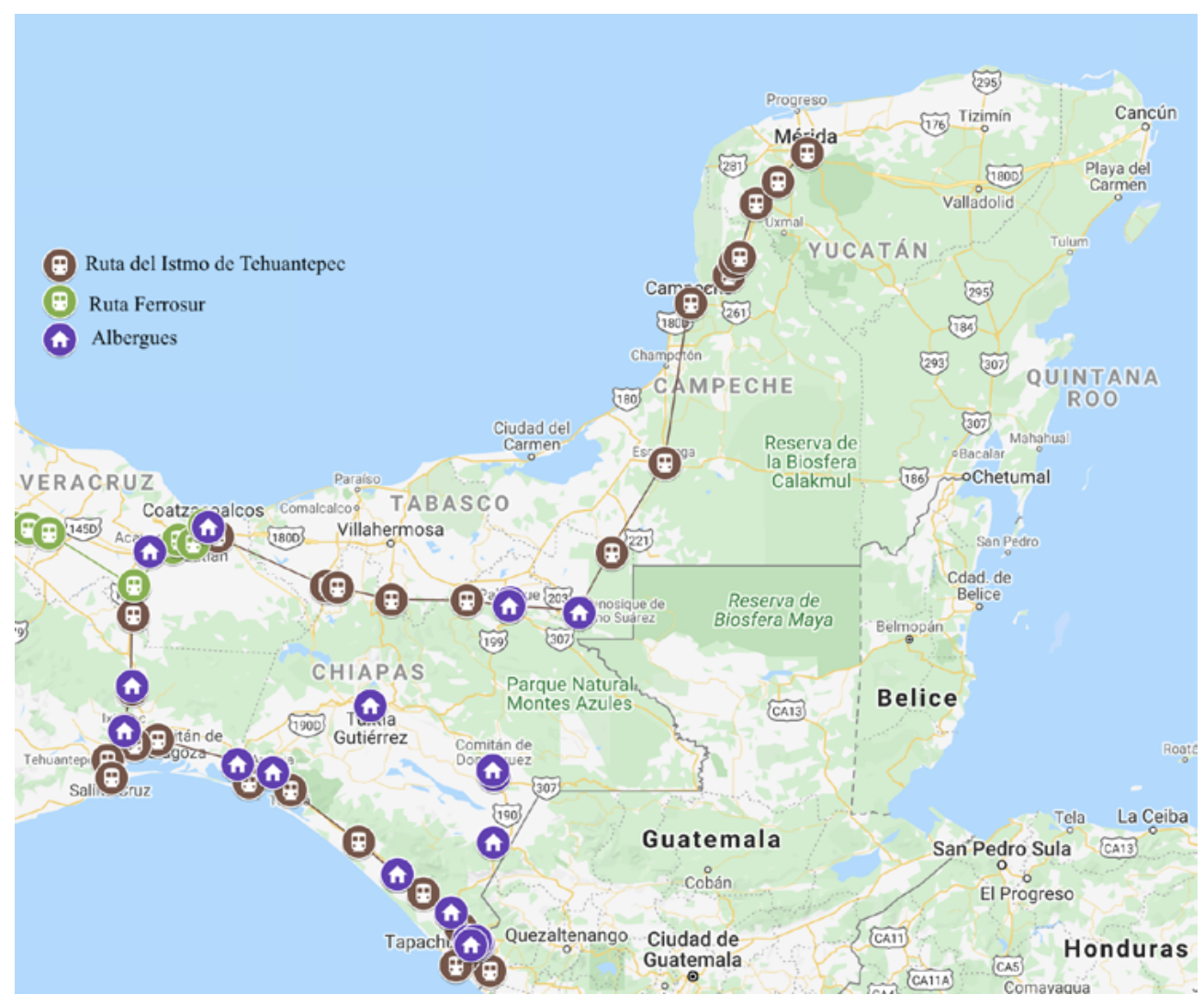

Fuente: Elaboración propia a partir de ARTF, 2020, OIM, 2018

Por otro lado, agrupamos con la etiqueta de actores de gobierno a organismos de la administración federal, estatal y local que enfocan sus actividades al control migratorio o a la política social o de salud, especialmente relevantes durante el periodo de pandemia. Las instituciones de gobierno desempeñan alternativamente actividades de control y registro de la migración, como pueden ser el INM y las estaciones migratoria bajo su dependencia, y la Comar dedicada al procesamiento de las solicitudes de refugio; también organismos de asesoramiento y diseño de políticas públicas en la materia, como la Unidad de Política Migratoria de la Secretaría de Gobernación, o bien otras unidades administrativas, de carácter estatal y/o local, abocadas a las políticas sociales o de salud. En su conjunto, se trata de actores fundamentales, constituidos sobre el principio del monopolio estatal de los medios legítimos de movimiento y de la capacidad determinante sobre las poblaciones nacionales y extranjeras (Torpey, 1998), pero que se les observa desoberanizados (Chignola, 2010; Negri, 2010) en un doble sentido: en primer lugar, frente al resto de los actores de la gobernanza, que influyen sobre manera en la gestión del fenómeno; en segundo lugar, por una 
evidente pérdida de control sobre la población migrante debido a la predominancia de su carácter irregular. Así lo reconoce uno de los agentes entrevistados dedicado al diseño de políticas públicas sobre migración del gobierno federal:

(...) uno de los elementos básicos en materia de política pública en los que habría que profundizar es en la regularización de los flujos migratorios. En tratar de que por lo menos los ingresos sean regulares, para saber quién entra y quién sale de tu país. (E-G02)

Aun así, a pesar de este déficit, en la Figura 2 observamos el despliegue copioso de estos actores junto a los albergues y a las vías del ferrocarril.

Figura 2: Albergues, sistema ferroviario y oficinas gubernamentales (estaciones migratorias, INM y Comar) en el istmo de Tehuantepec, México

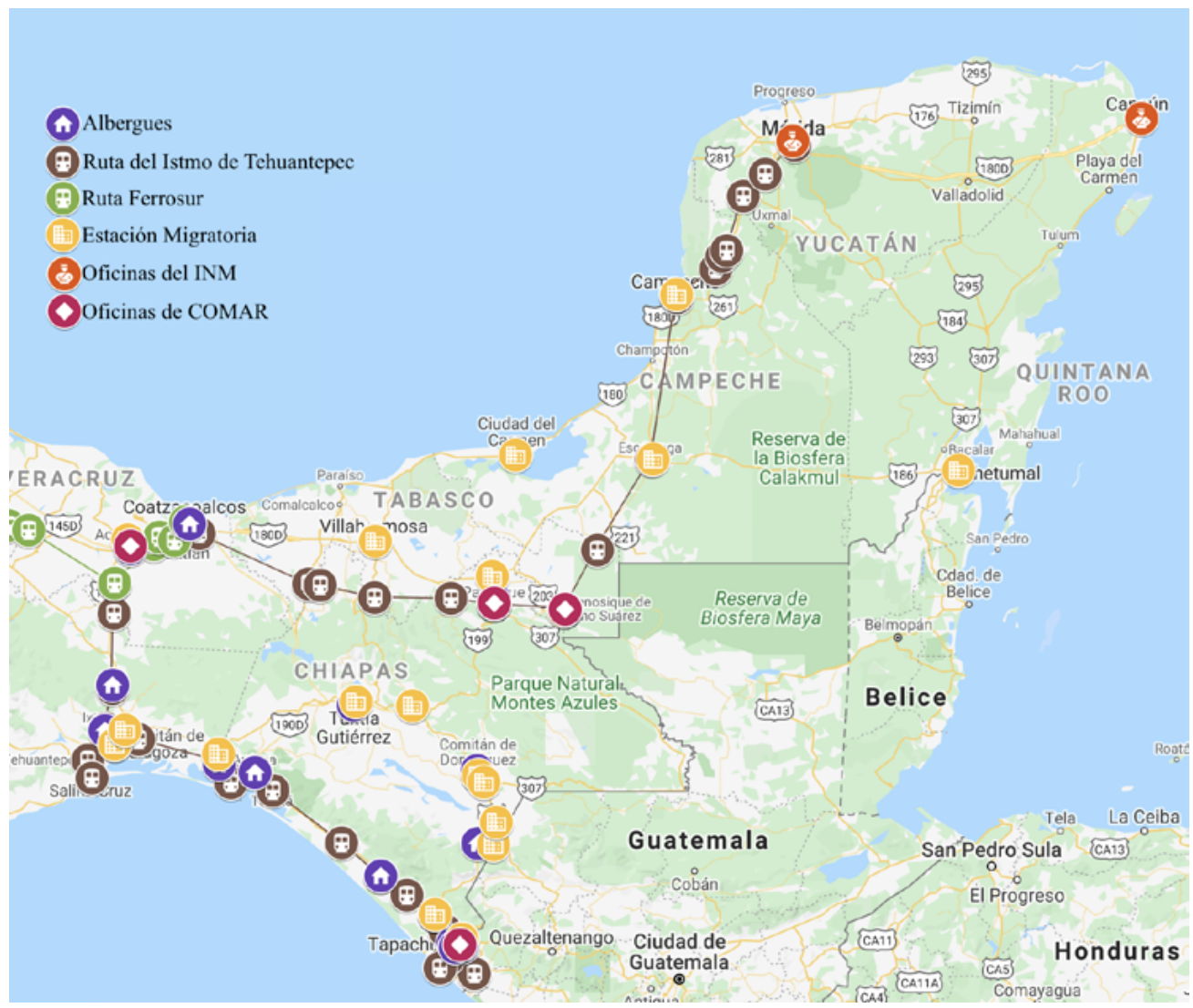

Fuente: Elaboración propia a partir de ARTF, 2020, Comar, 2020b, Global Detention Project, s.f., INM, s. f., OIM, 2018

Finalmente, entendemos por organismos internacionales aquellos que forman parte de agencias intergubernamentales (OIM, UNICEF y ACNUR), así como organismos de la sociedad civil internacional (Médicos Sin Fronteras [MSF]) y que atienden el tema de migración en México, tanto a partir de la gestión y documentación de la población migrante, como a través de la incidencia y la atención humanitaria. A través de la 
gestión cotidiana de la migración, estos actores reproducen valores de la gobernanza en el terreno. Encarnan principalmente sus aspectos normativos.

Si bien estos organismos no son nuevos en el país, es en años recientes que han crecido exponencialmente, expandiendo su representación en el territorio con oficinas "de terreno". La noción de oficina de terreno forma parte del lenguaje cotidiano de las organizaciones y da cuenta de un cambio que transita de un modelo de gestión burocrática enfocado a la incidencia sobre las políticas públicas gubernamentales, a otro en el que la atención a esta población es una prioridad.

Organismos como ACNUR, UNICEF, OIM y MSF han visto un importante crecimiento en sus operaciones a partir de las caravanas de 2018 y aquellas que siguieron, abrieron oficinas en la frontera norte y sur, y se aliaron con diferentes casas de migrantes para incrementar su alcance. A través de mandatos y de la capacitación y profesionalización de socios (los albergues y las organizaciones de la sociedad civil [osc]), los organismos internacionales han ido aumentando su poder en la coordinación de esta estructura de la gobernanza. Ejemplo de ello es Programa Casa Refugiados, una osc socia del ACNUR que desde 2015 se dedica a la asistencia humanitaria de los solicitantes de asilo y refugiados en Ciudad de México. Estos socios representan, para los organismos internacionales, un apoyo para la atención de una población que va en aumento, además de la posibilidad de habilitar espacios de atención en lugares donde no cuentan con una presencia operativa. Tanto el ACNUR COMO OIM, UNICEF y MSF tienen presencia en la red de albergues, ya sea a través de una oficina en la misma casa de migrantes, o por medio de actividades y visitas (MSF, 2020). La Figura 3 muestra la convergencia entre la infraestructura social que proporcionan los albergues o la logística de determinadas osc en la disposición de sus oficinas junto a las casas de migrantes.

Figura 3: Albergues en México y organismos internacionales (ACNUR, OIM, UNICEF, MSF)

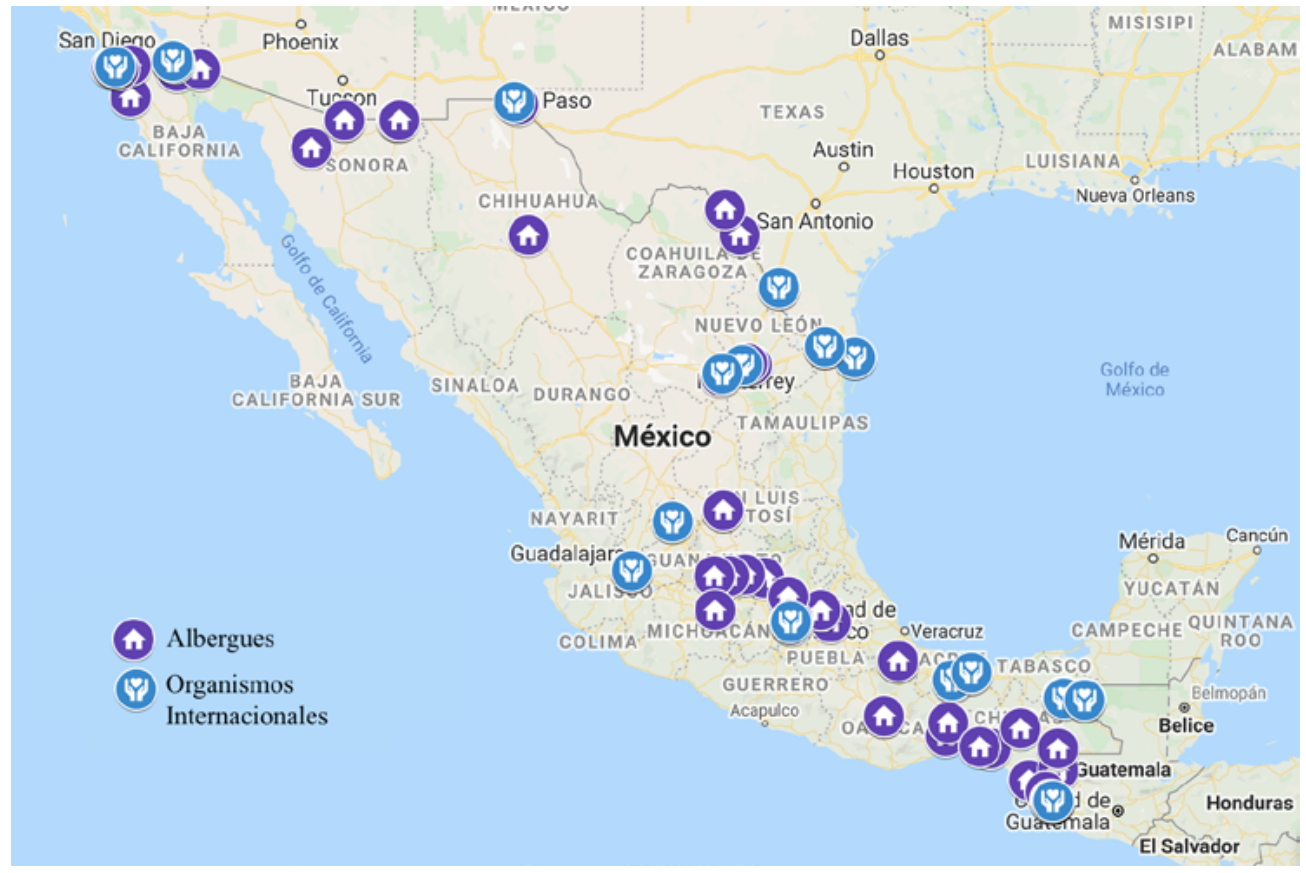

Fuente: Elaboración propia a partir de ACNUR, s.f., MSF, 2020, OIM, 2018 
El conjunto de estos actores hace al universo de nuestra investigación. En él observamos un espacio social abigarrado, descentralizado, heterogéneo y de relaciones de poder dispersas que, como puede verse en opinión de alguno de los agentes, es valorado por su escasa eficacia, aunque el déficit no se atribuye a cuestiones estructurales de la gobernanza, a sus principios y normas de funcionamiento, sino a una mala o incompleta aplicación de los mismos:

(...) como país no hemos logrado una definición clara, puntual, concisa o consistente, sobre la migración (...) la movilidad humana ¿no?, y en ese sentido los esfuerzos que existen, pues están muy desarticulados y no contribuyen a paliar las complejas situaciones que atraviesan las personas en movilidad, sean mexicanas o extranjeras, sean internas o externas. (...) Me parece que todavía estamos en un modelo donde la gobernanza no ha terminado de madurar. (E-G01)

En este denso panorama de actores e intervenciones sobre el fenómeno migratorio, la sociedad civil destaca a través de la marcada presencia de las casas de migrantes. El discurso y la cultura de los derechos humanos les asiste, a veces, cuando se tiene en cuenta a los organismos defensores de los derechos de los migrantes, de un modo más combativo y dirigido a la denuncia. ${ }^{3}$ Frente a ello, las instituciones de gobierno se constituyen como una estructura más o menos paralela que interviene sobre el reconocimiento de la irregularidad de los migrantes que atraviesan la frontera e, implícitamente, de la sociedad civil que los acoge. Como si la excepción fuera la norma (Gatti et al., 2021), los agentes de gobierno actúan a sabiendas de las fallas en los mecanismos de control monopólico de los medios autorizados de movilidad. Se agrega a ello el despliegue de las organizaciones internacionales que adquiere especial relevancia cuando el ACNUR comienza a intentar asir a las poblaciones migrantes a través de la figura del refugio, especialmente con el advenimiento de las caravanas migrantes en 2018. De todo ello, de este ingente despliegue de actores, en el campo de la gobernanza y en el espacio practicado que muestran los mapas, lo que destaca es la base social que representan las casas de migrantes. A estas concurren las organizaciones internacionales para desplegar su acción; frente a ellas se van posicionando los organismos gubernamentales; también a su lado despliegan su labor otras organizaciones de la sociedad civil que actúan en defensa de los derechos migrantes o denuncian sus condiciones de existencia. Las casas de migrantes instituyen la condición de tránsito del tipo de migración que es objeto de la gobernanza.

Es justamente esa base social la que se ha visto severamente afectada durante la pandemia repercutiendo en el conjunto de la gobernanza. Una de esas afectaciones tiene que ver con la dotación presupuestaria y de recursos de los albergues. Por un lado, por una afectación estructural, que tiene que ver con la suspensión de subvenciones que muchos de estos lugares reciben a través de la cooperación internacional de Estados Unidos. Por otro, se paralizó también la estructura local de apoyo de estos lugares: “(...) antes recibían recursos, por ejemplo, de una parroquia, y ahora por ejemplo, pues como las parroquias se cerraron, pues disminuyó también toda la ayuda" (E-SC5).

\footnotetext{
${ }^{3}$ Ver Informe sobre los efectos de la pandemia de COVID-19 en las personas migrantes y refugiadas, 2020; Informe alternativo de las organizaciones de la sociedad civil de México al Comité Contra la Tortura en la ONU 2012-2019, 2019.
} 
Además, la capacidad de atención de los albergues se vio mermada por la falta de personal, lo que llevó a que algunos de ellos tuvieran que cancelar completamente sus actividades:

(...) nosotros trabajamos solo con voluntarios, teníamos a un voluntario alemán del programa Fs y a una voluntaria de la iglesia luterana norteamericana, cuando empezó la pandemia en marzo pues sus organizaciones lo que hicieron fue sacarlos inmediatamente del país. (E-SC26)

Para una realidad social caracterizada fundamentalmente por el hacinamiento que representan estos lugares, la pandemia significó una amenaza letal. El cierre fue la realidad a la que se vieron abocados muchos de los albergues:

En marzo cerramos completamente, hablamos con las personas, teníamos en ese momento 30 personas. Y entonces les comentamos que por seguridad teníamos que cerrar y que ellos tenían obviamente la libertad de salir si querían hacerlo pero que pues ya no podían salir a trabajar. (E-SC26)

Desprotección sobre desprotección. Frente al cierre de estos establecimientos cabe suponer una nueva realidad de intemperie (Schindel, 2020). Si la realidad que observamos previa a la pandemia podría caracterizarse como de una producción política del abandono que deja expuesta a la población migrante a la acechante realidad del afuera (Gatti et al., 2021), las medidas de cierre de los albergues y la necesidad de liberar a la población migrante de las estaciones migratorias ${ }^{4}$ multiplican esa realidad:

La situación que atraviesan los migrantes es muy complicada, ya que a menudo se encuentran sin un lugar donde dormir, sufriendo enfermedades en el camino y enfrentándose al cierre de las fronteras en el norte y el sur del estado. (E-SC10)

Considerada la situación en su conjunto, el cambio que se produjo fue mayúsculo: las limitaciones a la libre circulación y las medidas de distanciamiento social dibujan un panorama de inmovilidad para una estructura montada sobre el principio de transitoriedad. Las estancias prolongadas modifican en muchos casos la esencia de los lugares, diseñados y dispuestos para el paso: “(...) si antes eran personas no identificadas que venían un día, ahora hemos sido familia, que hemos estado cinco meses compartiendo la vida juntos, ¿no?” (E-SC20).

Mientras que en su mayoría los albergues ven reducidas sus actividades y capacidades, en algunas regiones se institucionalizan albergues "espontáneos", como el Campamento de Matamoros, del cual el párroco que lo asiste relata cómo organizaciones como MSF o las propias autoridades sanitarias del Estado han oficializado su atención en las instalaciones; el ACNUR dice que ha incorporado nuevos socios a cuenta de la pandemia; otras organizaciones del sector salud se dedican a la atención de migrantes, como algunas de las secretarías del ramo de los gobiernos estatales. Es de destacar en este aspecto, las modificaciones en la actividad de los consulados, que se han dedicado

\footnotetext{
${ }^{4}$ Para dar seguimiento a las recomendaciones de protocolos sanitarios ante la pandemia, a finales de marzo, el INM comenzó a liberar a personas migrantes de las estaciones migratorias; a principios de este mes había 3759 personas en las estaciones, para finales de abril solo se tenían registradas 106 (INM, 2020). Ver también Informe sobre los efectos de la pandemia de COVID-19 en las personas migrantes y refugiadas, 2020.
} 
a desarrollar atención social hacia sus connacionales o a atender solicitudes de regreso a sus países de origen.

Como en su momento las caravanas, que significaron un revulsivo para la gobernanza de la migración en el país, la pandemia no fue de efecto menor en este sentido. Ha acentuado una de las propiedades esenciales de la gobernanza: la concepción de la realidad migratoria como crisis. Excepción sobre excepción: sobre la esencia de movilidad de su objeto de gobierno deviene el mandato de quietud; sobre la realidad del hacinamiento, las medidas de distanciamiento físico. Y ello desconcierta a todos sus actores: estancias de larga duración para los albergues; liberación de las estaciones migratorias; imposibilidad de virtualidad para operaciones de terreno; desconcierto en las redes de filantropía y desmantelamiento de la estructura del voluntariado en las organizaciones de la sociedad civil. A aquellos mapas de transitoriedad inestable, la pandemia les ha devuelto la quietud. Como si la excepción de la excepción restaurase la vieja cartografía en su vocación de dar cuenta de un orden de lugares estables y como si la esencia de movilidad y transitoriedad de los mapas de la primera excepción quedara suspendida con esta segunda excepción. Pero no. La pandemia se sobrepone como otra de las crisis que hace a la gobernanza de la migración.

\section{Identificar}

Una de las principales atribuciones de los estados nacionales es la determinación de quién es nacional y quién extranjero y, por lo tanto, de quién está "adentro" y quién "afuera" de pertenencia a la sociedad nacional, lo que garantiza a los primeros el acceso a los derechos y excluye de ello a los segundos. Lo que posibilita esta capacidad determinante es el progresivo desarrollo desde el siglo xix de grandes aparatos de identificación de las personas, dispositivos que hacen a los individuos irreductibles en la correspondencia de su cuerpo con un nombre (Gatti, 2008). Esa capacidad productora de individuos es atribución exclusiva de los Estados. Se relaciona con la tendencia a "abrazar" a sus poblaciones y con el ya mencionado monopolio sobre los medios legítimos de movimiento. Pasaportes y carnets de identidad son los documentos que habilitan la entrada, la salida y la pertenencia a una sociedad nacional. Identificar es, pues, atributo exclusivo y fundamental de la forma típica ideal de la organización política moderna llamada Estado (Torpey, 1998, 2020).

Identificar es también el otro gran verbo del campo de la gobernanza de la migración en México. Es la acción de la mayoría de sus actores. Casi todos los agentes identifican y registran. Lo hacen en cada albergue. De forma reiterada, a lo largo de la travesía, cada casa de migrante registra las entradas y salidas y recoge datos de las personas que ingresan según el criterio del propio establecimiento y desde la buena fe sobre lo que los migrantes declaran. También lo hacen los agentes de gobierno, en cada estación migratoria, en las oficinas del INM y de la Comar, en cada solicitud de refugio. Y lo hacen los organismos internacionales: el ACNUR en cada entrevista de atención o de entrega de apoyos económicos a personas refugiadas, en sus campamentos espontáneos que acompasan el paso de las caravanas, o nuevamente en cada casa de migrante donde despliegan sus burocracias; o la oim en los ejercicios de monitoreo de los flujos migratorios durante las caravanas de 2018. En la gobernanza de la migración no hay una instancia de identificación, hay varias. Y varias habrá de suponerse que son también las identidades que puede portar un mismo migrante 
calibrando estratégicamente de decirse una persona en un lugar y otra en otro en orden a proteger su integridad física al atravesar un territorio jalonado de violencias (Gatti \& Irazuzta, 2019; Gatti et al., 2021; Varela, 2020).

La falla en el control monopólico de los medios de movimiento del Estado es lo que explica que no haya una identidad, sino muchas; que no haya un registro, sino varios. La identificación multiplicada hace al dato de ese tipo de "migración de tránsito" (Oficina del Alto Comisionado para los Derechos Humanos [OACDH], 2018) esquivar a las categorías administrativas de reconocimiento de los no nacionales en un país; cercana a la condición de apátrida; no siempre en correspondencia con el estatus de refugiado; tampoco migrante a secas, como quien dice "migrante económico"; quizá desplazado, quizá migrante forzado. La condición migrante se multiplica en una variedad de categorías administrativas en la que la figura del refugio tiende a convertirse en hegemónica porque se pretende que contrarreste lo que de inasible tiene la condición de tránsito: “(...) cuando la persona solicita la condición de refugiado hay un plan de permanencia y un plan de una intención de integrarse, por lo que la atención debe ser enfocada a eso (...)" (E-OI2).

En esa política decidida de propiciar la integración a través del refugio se institucionaliza la condición excepcional de la migración. La vulnerabilidad es la clave para ese reconocimiento y la indagación en ella es la vía a través de la cual identifica el ACNUR y certifica la Comar. Quienes no pueden dar cuenta de esta condición o quienes deciden no darla, parecen quedar en un limbo de "normalidad". Despojados de condición excepcional, pareciera no haber reconocimiento:

Hay una discriminación estructural entre migrantes y refugiados. Se les da preferencia a los refugiados y no se les da preferencia al migrante normal (...) Le vas a dar preferencia al refugiado porque es el que tiene las estructuras, todo bien formadito, todo bien bonito, y al migrante ahí lo dejas abrazado de un poste. Por temas presupuestales, pero también de reconocimiento legal. (E-SC16)

Pero sobre todas las categorías prevalece una condición, la de ser una migración a la que se le presupone el tránsito, bien para acompasarlo, bien para detenerlo. "En tránsito" revela el movimiento $\mathrm{y}$, a la vez, la incapacidad de registrar ese movimiento como un orden estable de mantenimiento de la identidad de quienes se mueven. La irregularidad o la clandestinidad parecen ser condición indisociable de la migración en tránsito, por lo menos es lo que se deduce de las palabras de un agente de gobierno dedicado al diseño de políticas en la materia:

(...) tener un registro de personas en tránsito (...) para el propio migrante le quitaría lo clandestino, ¿no? Sería identificable, sería quizá hasta ubicable, ¿me explico? (...) Siempre se está hablando de que hay migración en tránsito a lo largo del país, por ejemplo, y que hay centenares, miles de personas migrantes que fallecieron en su tránsito por México, es porque jamás han sido identificadas. Es como si no hubieran pasado, como si no hubieran entrado. Deberíamos tener un registro, pero tener un registro también las haría totalmente identificables, ¿me explico? (E-G02)

"No contados" (Gatti et al., 2020), "desaparecidos sociales" (Gatti, 2020), "ilegales" (De Genova, 2002) o "sujetos imposibles": "una realidad social que es a la vez que una imposibilidad legal” (Ngai, 2005, p. 4). Decir migración en la realidad contemporánea 
de México es decir excepción, es dar cuenta de una situación en la que el hecho se sobrepone al derecho. Esa excepción no es ausencia de derecho entendido como norma. Las normas abundan; casi tanto como las acciones de identificación. La propia excepción parece ser la norma (Agamben, 2004; Ong, 2006); la propia gobernanza se desenvuelve normativamente sobre la excepción. Su dato estructural es el movimiento de poblaciones, la movilidad humana; el tránsito. Y todo ello entendido como "problema", gestionado desde un diagnóstico de crisis. Sobre ello se despliega el actuar de la gobernanza en ejercicios de gobierno que tienden a mermar el fallo en el control monopólico de los medios de movimiento y de la capacidad exclusiva de identificación sobre las personas que se mueven en una travesía a la que parece haberles advenido una valla infranqueable, bien porque algunos de esos migrantes entran en otra categoría, la que establece el programa MPP; $;{ }^{5}$ bien porque la pandemia ha venido a afectar al dato estructural de la gobernanza, el tránsito.

Con la pandemia, al movimiento le devino la quietud. Frente a las limitaciones a la libre circulación, la acción fundamental de identificación pierde su carácter prioritario. Así lo reconocen desde la Redodem (Red de Documentación de las Organizaciones Defensoras de Migrantes), la agrupación de casas de migrantes del Servicio Jesuita a Migrantes, que procura estandarizar los mecanismos de registro y documentación:

(...) [Con la pandemia] lo principal era poder brindar la atención humanitaria ¿no?, lo que los albergues hacen, pero no es la única labor, porque el motivo de que estén en la red justo es para registrar y documentar, pero realmente vimos que la necesidad prioritaria era poder tener suficientes recursos para poder seguir brindando la atención humanitaria y por eso fue que la apuesta los primeros meses fue en eso y no tanto en la apuesta del registro y de la documentación. (E-SC5)

Frente a la situación de paralización del movimiento, los no contados comienzan a dar cuenta de sí, los desaparecidos parecen aparecer e incluso los refugiados insinúan querer renunciar a tal condición. En el consulado de algún país centroamericano de la zona noreste de México no evitan críticas al ACNUR, pero para hacerlo piden parar la grabación. Dicen entonces que la condición de refugiado es la peor. Que quienes allí entran ingresan al desamparo total. Que todo el mundo se desentiende desde que se declara a alguien refugiado; que nadie atiende las necesidades de las personas refugiadas. Incluso, que eso inhibe la actuación del consulado porque el refugio supone que las personas pasan a estar bajo la tutela del Estado y del seguimiento del ACNUR, pero que nada de eso sucede. Cuentan que en esas situaciones todo lo que pueden hacer es mandarlos a CM, un albergue de la ciudad, porque son ellos quienes tienen un acuerdo con el AGNUR, pero que aun así les siguen llegando quejas.

Excepción de la excepción. La pandemia ha dejado en suspenso las múltiples y copiosas prácticas de identificación; bien porque la quietud inhibe nuevos ingresos a los albergues o porque estos mismos priorizan otro tipo de actividades justificadas en nuevas necesidades; bien porque se paralizan las actividades administrativas o se cortan o reducen los flujos migratorios. La pandemia muestra incluso el reverso de las categorías administrativas y clasificadoras de una misma población migrante. La migración deviene ahora una población homogénea, reunida bajo el imaginario

${ }^{5} \mathrm{Al}$ seguir lo establecido por los Protocolos de Protección de Migrantes (MPP por sus siglas en inglés), en 2019, 84000 migrantes habrían sido retornados a México después de haber solicitado asilo en Estados Unidos (Guzmán, 2020). 
consolidado de la intemperie y el tránsito: una representación de la desdicha, de la vulnerabilidad extrema y de la exposición a lo peor. Sobre todo, ahora, con las restricciones a la movilidad y la distancia física — dos cosas extrañas, si no opuestas, a lo que aquí se analiza—, la migración se representa como especialmente expuesta al virus y como fuente de contagio: "Se empezó a generar un discurso de migrante igual a persona con pandemia, y así. No sabes de dónde viene, no sabes con quién ha interactuado y todo eso, y se empieza a generar ese temor" (E-SC16).

El "problema" y la crisis de la gobernanza han mutado: si sus dispositivos de identificación iban dirigidos a conjurar lo extraño a la comunidad haciendo de algún modo asequible a una población en tránsito esquiva a los medios de movimiento legítimos, en situación de pandemia y bajo el mandato de quietud, el conjuro se cierne sobre la intemperie (Schindel, 2020), sobre la indigencia:

Nos enfocamos tanto en el desempleo, nos enfocamos tanto en el ¿Quédate en casa! Que ellos que no la tienen, los dejamos fuera. Los dejamos fuera. (...) Sí, se queda completamente varado y si no responde y si la suerte no le favorece, estamos hablando de un indigente más. (E-SC8)

\section{Reflexiones finales}

La excepción de la excepción no da como resultado una restauración de la normalidad, como si las instituciones políticas recuperaran las capacidades que se le presumen, como si los estados-nación recobrasen los controles monopólicos sobre la gestión de "sus" poblaciones en "sus" territorios, como si ese tipo ideal de organización política moderna pasara a corresponderse plenamente con la realidad. Casi todos los agentes que fueron entrevistados reconocen que la pandemia ha venido a excerbar los desórdenes y las vulnerabilidades ya existentes; ese reconocimiento seguramente se deba a la especial condición de visibilidad que esta propicia. Lo que en tales circunstancias se ve es un territorio atravesado por poblaciones que lo habitan en el tránsito y una gestión de esas poblaciones que las acompasa en un reconocimiento inestable y precario de los individuos que componen esas poblaciones.

No hay un futuro previsible para esas poblaciones ni para las instituciones que las gobiernan después de la pandemia; lo que si acaso se observa es que en ese cruce de población y territorio se dirimen las grandes cuestiones que sostienen a las instituciones políticas de la modernidad, al Estado-nación y a su correlación individual, la ciudadanía. En la encrucijada de esas dos grandes cuestiones, allí donde radica la crisis que gestiona la gobernanza de la migración, quizá se estén fraguando los cambios que conduzcan a una sociedad diferente. Por lo pronto, lo que se observa es que, a pesar del colosal esfuerzo de gobernar la migración, esta resulta en gran medida ingobernable.

\section{Referencias}

Agamben, G. (1998). Homo sacer. El poder soberano y la nuda vida. Pre-Textos.

Agamben, G. (2004). Estado de excepción: Homo sacer II, 1. Pre-Textos. 
Agencia Reguladora del Transporte Ferroviario (ARTF). (2020). Sistema Ferroviario Mexicano [mapa digital]. http://artf.centrogeo.org.mx/mviewer/sfm

Alto Comisionado de Naciones Unidas para los Refugiados (ACNUR). (s. f.). Contacta al ACNUR. https://help.unhcr.org/mexico/donde-encontrar-ayuda/contacta-al-acnur/

Arendt, H. (1960). Freedom and politics: A lecture. Chicago Review, 14(1), 28-46. http://www.jstor.org/stable/25733551

Bauder, H. (2016). Migration Borders Freedom. Routledge.

Betts, A. (2011). Global Migration Governance. Oxford University Press.

Bourdieu, P. (1997). Razones prácticas: sobre la teoría de la acción. Anagrama.

Chignola, S. (2010). A la sombra del Estado, governance, gubernamentalidad, gobierno. En C. Altamira (Comp.), Política y subjetividad en tiempos de governance. Waldhuter Editores.

Comisión Mexicana para la Ayuda a Refugiados (Comar). (2020a). Estadística Octubre 2020. https://www.gob.mx/comar/articulos/estadistica-octubre-2020?idi$\mathrm{om}=\mathrm{es}$

Comisión Mexicana para la Ayuda a Refugiados (Comar). (2020b). ¿¿Dónde está Comar? https://www.gob.mx/comar/articulos/donde-esta-comar?idiom=es

De Certeau, M. (2000). La invención de lo cotidiano: Artes de hacer. Universidad Iberoamericana.

De Genova, N. (2002). Migrant "illegality" and deportability in every day life. Annual Review of Anthropology, 31, 419-447. https://doi.org/10.1146/annurev.anthro.31.040402.085432

Doering-White, J. (2018). Evidencing violence and care along the Central American Migrant Trail through Mexico. Social Service Review, 92(3), 432-469. https:// www.journals.uchicago.edu/doi/10.1086/699196

Domenech, E. (2018). Gobernabilidad migratoria: producción y circulación de una categoría de intervención política. Revista Temas de Antropología y Migración, (10), 110-118. https://ri.conicet.gov.ar/bitstream/handle/11336/93417/ CONICET_Digital_Nro.5bd8f470-2f65-4f45-8a6c-68e354112a18_X.pdf?sequence $=5$ \&isAllowed $=\mathrm{y}$

Fassin, D. (2007). Humanitarianism: a nongovernmental government. En M. Feher, Nongovernmental Politics (pp. 149-60). Zone Books.

Fassin, D. (2012). Humanitarian reason: a moral history of the present. University of California Press.

Fassin, D. (2018). Por una repolitización del mundo. Las vidas descartables como desafío del siglo XXI. Siglo XXI.

Finkelstein, L. (1995). What is global governance? Global governance, 1(3), 367-372. http://www.jstor.org/stable/27800120

Foucault, M. (1980). La gubernamentalidad. En AA. VV. La microfísica del poder. Ediciones de la Piqueta.

Gatti, G. (2008). El detenido desaparecido. Narrativas posibles para una catástrofe de la realidad. Ediciones Trilce.

Gatti, G. (2017). Un mundo de víctimas. Antropos. 
Gatti, G. (2020). The social disappeared: genealogy, global circulations, and (possible) uses of a category for bad life. Public Culture, 32(1), 25-43. https://www. researchgate.net/publication/338810741_The_Social_Disappeared_Genealogy_Global_Circulations_and_Possible_Uses_of_a_Category_for_the_Bad_Life

Gatti, G., Irazuzta, I. \& Martínez, M. (2021). Inverted exception. Ideas for thinking about the new disappearances through two case studies. Journal of Latin American Cultural Studies, 29(4), 581-604. https://doi.org/10.1080/13569325.2020.1 839869

Gatti, G., Irazuzta, I. \& Sáez, R. (2020). Los no contados. Desbordamientos del concepto jurídico de desaparición. Athenea Digital, 20(3), 1-17. https://atheneadigital. net/article/view/v20-3-gatti-irazuzta-saez

Gatti, G. \& Irazuzta, I. (2019). Diario de la desaparición mexicana. Entre el precedente y el exceso. Disparidades. Revista de Antropología, 74(2). https:/ / doi.org/10.3989/ dra.2019.02.019

Global Detention Project. (s. f.). Mexico (country profile). https://www.globaldetentionproject.org/countries/americas/mexico

Guzmán, S. (2020). EU regresó a México 84 mil migrantes que solicitaron asilo en 2019. https://www.elfinanciero.com.mx/nacional/en-un-ano-estados-unidos-regreso-a-mexico-84-mil-migrantes-que-esperan-por-asilo/

Informe alternativo de las organizaciones de la sociedad civil de México al Comité Contra la Tortura en la oNU 2012-2019. (2019). http://cmdpdh.org/project/informe-alternativo-de-las-organizaciones-de-la-sociedad-civil-de-mexico-al-comite-contra-la-tortura-de-la-onu-2012-2019/

Informe sobre los efectos de la pandemia de COVID-19 en las personas migrantes y refugiadas. (2020). http://www.cmdpdh.org/publicaciones-pdf/cmdpdh-informe-migracion-y-covid-19.pdf

Instituto Nacional de Migración (INM). (2020). Actúa INM con responsabilidad ante la contingencia por COVID-19 (Boletín No. 125/2020). https:/ /www.gob.mx/inm/prensa/ actua-inm-con-responsabilidad-ante-la-contingencia-por-covid-19-241034\#: :text $=$ Ante $\% 201 \mathrm{a} \%$ 20emergencia $\% 20$ sanitaria $\% 20$ actual, $\mathrm{a} \% 20$ plenitud $\% 20$ sus\%20derechos\%20humanos

Instituto Nacional de Migración (INM). (s.f.). Directorio. https://www.gob.mx/cms/uploads/attachment/file/454892/DIRECTORIO_Oficinas.pdf

Irazuzta, I. \& Martínez, M. (Coords.). (2014). De la identidad a la vulnerabilidad. Bella Terra.

Kooiman, J. (1993). Modern governance. New government society interactions. Sage Publications.

Launay, C. (2005). La gobernanza: Estado, ciudadanía y renovación de lo político. Origen, definición e implicaciones del concepto en Colombia. Revista Controversia, (185), 92-105. http://bibliotecavirtual.clacso.org.ar/Colombia/ cinep/20100925104922/lagobernanzaControversia185.pdf

Lippert, K. \& Rehaag, S. (2013). Sanctuary practices in international perspectives. Migration, citizenship and social movements. Routledge.

Mayntz, R. (2000). Nuevos desafíos de la teoría de Governance. Instituciones y Desarrollo, 7.

Mbembe, A. (2011). Necropolítica, seguido de sobre el gobierno privado indirecto. Melusina. 
Médicos Sin Fronteras (MSF). (2020). Mapa ruta migrante. https://www.msf.mx/sites/ mexico/files/-nuevo_map_ruta_migrante.pdf

Mezzadra, S. (2005). Derecho de fuga. Migraciones, ciudadanía y globalización. Traficantes de sueños.

Mezzadra, S. (2020). What happens to freedom of movement during a pandemic? Open Democracy. https://www.opendemocracy.net/en/can-europe-make-it/ what-happens-freedom-movement-during-pandemic/

Mezzadra, S. \& Neilson, B. (2013). Border as method, or, the multiplication of labor. Duke University Press.

Natera Peral, A. (2005). Nuevas estructuras y redes de gobernanza. Revista mexicana de sociología, 67(4), 755-791. http://www.scielo.org.mx/scielo.php?script=sci_arttext\&pid=S0188-25032005000400004\&lng=es\&tlng=es

Negri, A. (2010). La soberanía, entre el gobierno, la excepción y la governance. En C. Altamira (Comp.), Política y subjetividad en tiempos de governance (pp. 333-360). Waldhuter Editore.

Ngai, N. (2005). Impossible subjects: illegal aliens and the making of modern America. Princeton University Press. https://www.jstor.org/stable/j.ctt5hhr9r

Oficina del Alto Comisionado para los Derechos Humanos (OACDH). (2018). Situación de los migrantes en tránsito. https://acnudh.org/situacion-de-los-migrantes-en-transito/

Organización Internacional para las Migraciones (OIM). (2015). Marco de gobernanza sobre la migración. https://publications.iom.int/es/system/files/migof_brochure_sp.pdf

Organización Internacional para las Migraciones (OIM). (2018). Directorio de casas y albergues para personas migrantes. https://kmhub.iom.int/sites/default/files/directorio_de_casas_y_albergues_para_personas_migrantes_digital_0.pdf

Organización Internacional para las Migraciones (OIM). (2019). Glosary on migration. International Migration Law, (34). https://publications.iom.int/system/files/ pdf/iml_34_glossary.pdf

Organización Internacional para las Migraciones (OIM). (2020, noviembre). Tendencias migratorias durante la COVID-19 en Centroamérica, Norteamérica y el Caribe. https:/ / rosanjose.iom.int/site/sites/default/files/Reportes/tendencias_migratorias_ durante_la_covid-19_en_centroamerica_norteamerica_y_el_caribe_-_oim_.pdf

Olvera, A. (Comp.). (2001). La sociedad civil: de la teoría a la realidad. Colegio de México. https://www.jstor.org/stable/j.ctv6mtcjq

Ong, A. (2006). Neoliberalism as exception. Mutations in citizenship and sovereignty. Duke University Press.

Pérez-Agote, A. (1989). Sociología del nacionalismo. Servicio Editorial Universidad del País Vasco.

Pierre, J. (2000). Introduction: understanding governance. En J. Pierre (Ed.), Debating Governance (pp.1-10). Oxford University Press.

Porras, F. (2007). Rethinking local governance: hierarchies and networks in Mexican cities. European Review of Latin American and Caribbean Studies, 83, 43-59. https:/ / www.jstor.org/stable/25676280?seq=1

Powell, W. \& DiMaggio, P. (1991). The new institutionalism in organizational analysis. University of Chicago Press. 
Sassen, S. (2006). Territory, authority, rights: from medieval to global assemblages. Princeton University Press. https://www.jstor.org/stable/j.ctt7sx98

Schindel, E. (2020). Desiertos, mares, islas: geografías de intemperie como espacios de desaparición en contextos migratorios. Papeles del CEIC, 2020(1), 1-16. https://addi.ehu.es/bitstream/handle/10810/47200/20909-83078-1-PB.pdf?sequence $=1$ \&isAllowed $=y$

Schmitt, C. (2009). Teología política. Trotta.

Shapiro, M. (2001). Administrative law unbounded: reflections on government and governance, Indiana Journal of Global Legal Studies, 8(2), pp. 369-377.

Shoemaker, K. (2013). Sanctuary for crime in the early common law. En R. Lippert \& S. Rehaag, International perspectives. Migration, citizenship and social movements. Routledge.

Slack, J. (2019). Deported to death how drug violence is changing migration on the Us-Mexico border. University of California Press.

Torpey, J. (1998). Coming and going: on the state monopolization of the legitimate "means of movement". Sociological Theory, 16(3), 239-259. http://www.jstor.org/ stable/202182

Torpey, J. (2020). La invención del pasaporte. Estado, vigilancia y ciudadanía. Cambalache. Urry, J. (2007). Mobilities. Polity.

Varela, A. (2019). Capitalismo caníbal: migraciones, violencia y necropolítica en Mesoamérica. En B. Cordero, S. Mezzadra \& A. Varela (Coords.), América Latina en movimiento. Migraciones, límites a la movilidad y sus desbordamientos. Traficantes de sueños.

Varela, A. (2020). Necropolitica y migración en la frontera vertical mexicana. Un ejercicio de conocimiento situado. UNAM. http://ru.juridicas.unam.mx/xmlui/han$\mathrm{dle} / 123456789 / 58574$

Washington, J. (2020). The dispossessed. A story of asylum at the us-Mexican border and beyond. Verso.

Ignacio Irazuzta

Argentino. Cuenta con un doctorado en sociología y ciencia política por la Universidad del País Vasco (UPV). Actualmente labora como profesor de teoría política del Departamento de Ciencia Política y Relaciones Internacionales del Tecnologico de Monterrey, Campus Monterrey. Es miembro del Sistema Nacional de Investigadores de México. Sus líneas de investigación exploran temas sobre exclusión y vulnerabilidad, además de migración internacional y diásporas. Su publicación más reciente es: Gatti, Irazuzta \& Martínez. (2021). Inverted exception. ideas for thinking about the new disappearances through two case studies. Journal of Latin American Cultural Studies, 29(4), 581-604. https://doi.org/10.1080/13569325.2020.1839869

Idalia Ibarra

México-americana. Cuenta con una maestría en resolución de conflictos por la Universidad de Essex en Inglaterra, así como una licenciatura en relaciones internacionales por el Tecnologico de Monterrey. Actualmente es candidata a doctora en ciencias sociales por el ITESM, Campus Monterrey. Su investigación doctoral se centra en el estudio del régimen del refugio en México. 